\title{
A Note on Lower Bounds for Non-interactive Message Authentication Using Weak Keys
}

\author{
Divesh Aggarwal * \\ Divesh. Aggarwal@epfl.ch
}

\author{
Alexander Golovnev ${ }^{\dagger}$ \\ alexgolovnev@gmail.com
}

\begin{abstract}
In this note, we prove lower bounds on the amount of entropy of random sources necessary for secure message authentication. We consider the problem of non-interactive $c$-time message authentication using a weak secret key having min-entropy $k$. We show that existing constructions using $(c+1)$-wise independent hash functions are optimal.

This result resolves one of the main questions left open by the work of Dodis and Spencer [2] who considered this problem for one-time message authentication of one-bit messages.
\end{abstract}

\section{Introduction}

\subsection{Non-interactive Message Authentication}

In this note, we revisit the problem of non-interactive message authentication: where Alice and Bob share a weak secret key $R \in\{0,1\}^{n}$, and Alice wants to communicate up to $c$ messages authentically to Bob over a channel controlled by the adversary Eve. This problem is known to have an easy solution with $\varepsilon$-security for $\varepsilon<1$ using one of various possible universal hash functions, or more generally $c+1$-wise independent hash functions (see, for example, $[6,5]$ that give construction for $c=1)$. These solutions, however, require that the min-entropy $\mathbf{H}_{\infty}(R)$ of the source $R$ is at least $\frac{c n}{c+1}+\log \left(\frac{1}{\varepsilon}\right)$.

Dodis and Spencer [2] studied this problem with the goal of finding a lower bound on the minentropy of $R$. They showed that for any integer $k \geq \frac{n}{2}$, and any one-round message authentication protocol for one-bit messages, there exists a $k$-flat source $R$ such that the advantage of the adversary in forging the tag is at least $2^{n / 2-k}$, or in other words, $\mathbf{H}_{\infty}(R) \geq \frac{n}{2}+\log \left(\frac{1}{\varepsilon}\right)$. This showed that the construction using universal hash functions is optimal for one-bit messages. However, the bound for many time message authentication is still far from optimal and this was left as one of the main open questions in [2]. Specifically, the authors state that it is interesting to extend their quantitative results for private-key encryption and especially authentication to larger than one-bit message spaces. While this question has subsequently been almost resolved for the case of privatekey encryption [1], it has remained open for the case of private-key authentication.

\footnotetext{
${ }^{*}$ Department of Computer Science, EPFL

${ }^{\dagger}$ New York University
} 


\subsection{Our contribution and Comparison with [2]}

We answer this open question in the affirmative, i.e., that for any integer $k \geq \frac{c n}{c+1}$, and any $c$ round message authentication protocol, there exists a $k$-flat source $R$ such that the advantage of the adversary in forging the tag is at least $2^{c n /(c+1)-k}$, or in other words, $\mathbf{H}_{\infty}(R) \geq \frac{c n}{c+1}+\log \left(\frac{1}{\varepsilon}\right)$. Our proof uses a simple idea based on the chain rule for Shannon entropy.

In comparison, the result of [2] was proved by considering a bipartite multigraph with the edges corresponding to the keys and the vertices on each part corresponding to the tags of the bit 0 and 1, respectively. They then partitioned their proof into two cases (i) where there are few tags corresponding to the bit 0 , in which case it is easy to guess $\operatorname{Tag}(0, R)$, and (ii) where there are many tags corresponding to the bit 0 , but where knowing $\operatorname{Tag}(0, R)$ gives significant information about $\operatorname{Tag}(1, R)$. It seems that one might be able to generalize this idea to prove a lower bound for $c$-time message authentication by considering $c+1$ cases as opposed to considering two cases for $c=1$. However, the case analysis becomes significantly more involved due to the combinatorial nature of the proof, and perhaps this is a reason why the question has remained open for so long.

\section{Preliminaries}

For a set $S$, we let $U_{S}$ denote the uniform distribution over $S$. For an integer $m \in \mathbb{N}$, we let $U_{m}$ denote the uniform distribution over $\{0,1\}^{m}$, the bit-strings of length $m$. For a distribution or random variable $X$ we write $x \leftarrow X$ to denote the operation of sampling a random $x$ according to $X$. For a set $S$, we write $s \leftarrow S$ as shorthand for $s \leftarrow U_{S}$.

\subsection{Entropy Definitions}

The prediction probability of a random variable $X$ is defined as

$$
\operatorname{Pred}(X):=\max _{x} \operatorname{Pr}[X=x]
$$

The min-entropy of $X$ is defined as

$$
\mathbf{H}_{\infty}(X):=-\log \operatorname{Pred}(X) .
$$

We say that a random variable $X$ is an $(n, k)$-source if $X \in\{0,1\}^{n}$ and $\mathbf{H}_{\infty}(X) \geq k$. We also define conditional prediction probability of a random variable $X$ conditioned on another random variable $Z$ as

$$
\begin{aligned}
\operatorname{Pred}(X \mid Z) & :=\mathrm{E}_{z \leftarrow Z}\left[\max _{x} \operatorname{Pr}[X=x \mid Z=z]\right] \\
& =\mathrm{E}_{z \leftarrow Z}\left[2^{-\mathbf{H}_{\infty}(X \mid Z=z)}\right] .
\end{aligned}
$$

The conditional min-entropy of $X$ is defined as

$$
\mathbf{H}_{\infty}(X \mid Z):=-\log \operatorname{Pred}(X \mid Z) .
$$

Also, the Shannon entropy $\mathbf{H}_{1}(X)$ of a random variable $X$ is defined as

$$
\mathbf{H}_{1}(X):=-\sum_{x} \operatorname{Pr}[X=x] \log \operatorname{Pr}[X=x] .
$$


The conditional Shannon entropy of a random variable $X$ conditioned on another random variable $Z$ is defined as

$$
\begin{aligned}
\mathbf{H}_{1}(X \mid Z) & :=\mathrm{E}_{z \leftarrow Z} \mathbf{H}_{1}(X \mid Z=z) \\
& =-\mathrm{E}_{z \leftarrow Z} \sum_{x} \operatorname{Pr}[X=x \mid Z=z] \log \operatorname{Pr}[X=x \mid Z=z] .
\end{aligned}
$$

We will need the following standard facts about (conditional) min-entropy, and (conditional) Shannon entropy.

Fact 1. Let $X, Y, Z$ be arbitrary random variables, and let $f$ be an arbitrary function. Then the following hold

1. $\mathbf{H}_{\infty}(X \mid Z) \geq \mathbf{H}_{\infty}(f(X) \mid Z)$, and $\mathbf{H}_{1}(X \mid Z) \geq \mathbf{H}_{1}(f(X) \mid Z)$.

2. $\mathbf{H}_{1}(X, Y \mid Z)=\mathbf{H}_{1}(X \mid Y, Z)+\mathbf{H}_{1}(Y \mid Z)$.

3. $\mathbf{H}_{1}(X \mid Z) \geq \mathbf{H}_{\infty}(X \mid Z)$.

We remark here that the definition of the conditional Shannon entropy is fairly standard, but there are other alternative definitions in the literature for conditional min-entropy. However, our proposed definition is by now fairly standard. We direct the reader to [3] which contains a comprehensive discussion on conditional entropies, and proves Fact 1 among several other results.

\subsection{Message Authentication Codes}

In order to define a message authentication code, we first introduce the following game $\mathrm{G}_{c}(r)$. For a given function Tag : $\mathcal{M} \times\{0,1\}^{n} \mapsto \mathcal{T}$ and a fixed secret key $r \in\{0,1\}^{n}$, an adversary Eve is allowed to make at most $c$ adaptive queries $\mu_{1}, \ldots, \mu_{c}$ to $\operatorname{Tag}(\cdot, r)$. We say that Eve wins the game if she outputs a pair $\left(\mu_{c+1}, \sigma\right)$, such that $\operatorname{Tag}\left(\mu_{c+1}, r\right)=\sigma$ and $\mu_{c+1} \notin\left\{\mu_{1}, \ldots, \mu_{c}\right\}$. We define the advantage of Eve in this game as

$$
\operatorname{Adv}_{c}^{\text {Eve }}(r)=\operatorname{Pr}\left[\text { Eve wins } G_{c}(r)\right] .
$$

Definition 1. A function Tag : $\mathcal{M} \times\{0,1\}^{n} \mapsto \mathcal{T}$ is called a c-time $(n, k, \varepsilon)$-secure message authentication code, if for any distribution $R$ on $\{0,1\}^{n}$ with $\mathbf{H}_{\infty}(R) \geq k$, for any computationally unbounded adversary Eve,

$$
\mathrm{E}_{r \leftarrow R}\left[\operatorname{Adv}_{c}^{\mathrm{Eve}}(r)\right] \leq \varepsilon .
$$

\section{$2.3 \quad k$-wise Independent Hash Functions}

Here we define and give a well-known construction of $k$-wise independent hash functions.

Definition 2. A function $H: \mathcal{X} \times \mathcal{R} \mapsto \mathcal{Y}$ is said to be a $k$-wise independent hash function if for all $y_{1}, \ldots, y_{k} \in \mathcal{Y}$, and all distinct $x_{1}, \ldots, x_{k} \in \mathcal{X}$,

$$
\operatorname{Pr}_{r \leftarrow \mathcal{R}}\left(H\left(x_{1}, r\right)=y_{1} \wedge \cdots \wedge h\left(x_{k}, r\right)=y_{k}\right)=\frac{1}{|\mathcal{Y}|^{k}} .
$$

Lemma 1 (folklore). Let $k$ be a positive integer, and let $\mathcal{X}=\mathcal{Y}=\mathbb{F}$, and $\mathcal{R}=\mathbb{F}^{k}$ for some finite field $\mathbb{F}$. Then the function $H: \mathcal{X} \times \mathcal{R} \mapsto \mathcal{Y}$ given by

$$
H\left(x,\left(r_{0}, \ldots, r_{k-1}\right)\right):=r_{0}+r_{1} \cdot x+\cdots+r_{k-1} \cdot x^{k-1}
$$

is a $k$-wise independent hash function. 


\section{Tight Bound for $c$-time MACs}

In this section, we prove a lower bound on the error-probability $\varepsilon$ for $c$-time message authentication protocol for deterministic functions Tag.

Theorem 1. Let Tag be a c-time $(n, k, \varepsilon)$-secure message authentication code where Tag : $\mathcal{M} \times$ $\{0,1\}^{n} \mapsto \mathcal{T}$. Then we have the following.

1. If $k \leq \frac{c n}{c+1}$ then $\varepsilon=1$;

2. If $k>\frac{c n}{c+1}$ then $\varepsilon \geq 2^{\frac{c n}{c+1}-k}$.

Proof. Let $U$ be an $n$-bit uniformly random string, and let $\mu_{1}, \ldots, \mu_{c+1} \in \mathcal{M}$ be fixed distinct messages. Note that $\mathbf{H}_{1}(U)=n$. Using Fact 1 multiple times, we get

$$
\begin{aligned}
n & =\mathbf{H}_{1}(U) \geq \mathbf{H}_{1}\left(\operatorname{Tag}\left(\mu_{1}, U\right), \ldots, \operatorname{Tag}\left(\mu_{c+1}, U\right)\right) \\
& =\mathbf{H}_{1}\left(\operatorname{Tag}\left(\mu_{1}, U\right)\right)+\mathbf{H}_{1}\left(\operatorname{Tag}\left(\mu_{2}, U\right), \ldots, \operatorname{Tag}\left(\mu_{c+1}, U\right) \mid \operatorname{Tag}\left(\mu_{1}, U\right)\right) \\
& =\ldots \\
& =\sum_{i=1}^{c+1} \mathbf{H}_{1}\left(\operatorname{Tag}\left(\mu_{i}, U\right) \mid \operatorname{Tag}\left(\mu_{1}, U\right), \ldots, \operatorname{Tag}\left(\mu_{i-1}, U\right)\right) \\
& \geq \sum_{i=1}^{c+1} \mathbf{H}_{\infty}\left(\operatorname{Tag}\left(\mu_{i}, U\right) \mid \operatorname{Tag}\left(\mu_{1}, U\right), \ldots, \operatorname{Tag}\left(\mu_{i-1}, U\right)\right) .
\end{aligned}
$$

Therefore, there exists $i \in\{1, \ldots, c+1\}$, such that

$$
\mathbf{H}_{\infty}\left(\operatorname{Tag}\left(\mu_{i}, U\right) \mid \operatorname{Tag}\left(\mu_{1}, U\right), \ldots, \operatorname{Tag}\left(\mu_{i-1}, U\right)\right) \leq \frac{n}{c+1} .
$$

We fix an $i$ satisfying this ineqaulity. For any $\mathbf{t}=\left(t_{1}, \ldots, t_{i-1}\right) \in \mathcal{T}^{i-1}$, let $\mathcal{E}(\mathbf{t})$ be a shorthand for the event that $\operatorname{Tag}\left(\mu_{j}, U\right)=t_{j}$ for $1 \leq j<i$. From the definition of conditional min-entropy, we get the following.

$$
\begin{aligned}
2^{-\frac{n}{c+1}} & \leq \mathrm{E}_{\mathbf{t} \in \mathcal{T}^{i-1}} \max _{t_{i} \in \mathcal{T}} \operatorname{Pr}\left[\operatorname{Tag}\left(\mu_{i}, U\right)=t_{i} \mid \mathcal{E}(\mathbf{t})\right] \\
& =\sum_{\mathbf{t} \in \mathcal{T}^{i-1}} \operatorname{Pr}[\mathcal{E}(\mathbf{t})] \cdot \max _{t_{i} \in \mathcal{T}} \operatorname{Pr}\left[\operatorname{Tag}\left(\mu_{i}, U\right)=t_{i} \mid \mathcal{E}(\mathbf{t})\right] \\
& =\sum_{\mathbf{t} \in \mathcal{T}^{i-1}} \max _{t_{i} \in \mathcal{T}} \operatorname{Pr}\left[\operatorname{Tag}\left(\mu_{j}, U\right)=t_{j} \text { for } 1 \leq j \leq i\right]
\end{aligned}
$$

For every fixed $\mathbf{t}=\left(t_{1}, \ldots, t_{i-1}\right) \in \mathcal{T}^{i-1}$, let $\mu_{\mathbf{t}}$ be the most probable value of $\operatorname{Tag}\left(\mu_{i}, U\right)$ given $\operatorname{Tag}\left(\mu_{j}, U\right)=t_{j}$ for $1 \leq j<i$. Intuitively, we want to choose a distribution over the set of keys so that $\operatorname{Tag}\left(\mu_{j}, U\right)=t_{j}$ for $1 \leq j<i$ implies that $\operatorname{Tag}\left(\mu_{i}, U\right)=\mu_{\mathbf{t}}$. Then, given tags for $\mu_{1}, \ldots, \mu_{i-1}$, we can always guess the tag for $\mu_{i}$. Let $\mathcal{K}_{\mathbf{t}}$ be the set of keys corresponding to $\mu_{\mathbf{t}}$, i.e.,

$$
\mathcal{K}_{\mathbf{t}}=\left\{r \in\{0,1\}^{n} \mid \operatorname{Tag}\left(\mu_{i}, r\right)=\mu_{\mathbf{t}}, \operatorname{Tag}\left(\mu_{j}, r\right)=t_{j} \text { for } 1 \leq j<i\right\} .
$$

Let also

$$
\mathcal{K}=\bigcup_{\mathbf{t} \in \mathcal{T}^{i-1}} \mathcal{K}_{\mathbf{t}}
$$


From inequality (1),

$$
|\mathcal{K}| \geq 2^{n} \cdot 2^{\frac{-n}{c+1}}=2^{\frac{c n}{c+1}} .
$$

If $2^{k} \leq|\mathcal{K}|$, then let $\mathcal{R}$ be an arbitrary $2^{k}$ element subset of $\mathcal{K}$. Otherwise, let

$$
\mathcal{R}=\mathcal{K} \cup \mathcal{K}^{\prime}
$$

where $\mathcal{K}^{\prime}$ is a set of arbitrary keys from the set $\{0,1\}^{n} \backslash \mathcal{K}$, such that $|\mathcal{R}|=2^{k}$.

We claim that if $R$ is uniformly distributed on $\mathcal{R}$, then there exists a strategy for Eve such that the advantage in guessing $\operatorname{Tag}\left(\mu_{i}, r\right)$ given $\operatorname{Tag}\left(\mu_{1}, r\right), \ldots, \operatorname{Tag}\left(\mu_{i-1}, r\right)$ is at least $2^{\frac{c n}{n+1}-k}$ if $k>\frac{c n}{n+1}$, and 1, otherwise. To see this, notice that for any $r \in \mathcal{K}$, there is a unique value of $\operatorname{Tag}\left(\mu_{i}, r\right)$ given $\operatorname{Tag}\left(\mu_{1}, r\right), \ldots, \operatorname{Tag}\left(\mu_{i-1}, r\right)$. Let the strategy of Eve be to guess this unique tag assuming $R \in \mathcal{K}$. Then, Eve succeeds with probability 1 if $R \in \mathcal{K}$, and hence the advantage of Eve is

$$
\varepsilon \geq \frac{|\mathcal{R} \cap \mathcal{K}|}{2^{k}} \geq \frac{\min \left(2^{k}, 2^{\frac{c n}{c+1}}\right)}{2^{k}} .
$$

The statement of the theorem now follows.

It is well-known that the bound from Theorem 1 can be achieved by using a family of $(c+1)$-wise independent hash functions (see [4] for similar results). For the sake of completeness, we present this construction below.

Lemma 2 (folklore). Let $\mathbb{F}$ be a finite field, and let $\mathcal{M}=\mathcal{T}=\mathbb{F}$, and let the set of keys be $\mathbb{F}^{c+1}$ with $n=(c+1) \log |\mathbb{F}|$. Then the function Tag: $\mathcal{M} \times \mathbb{F}^{c+1} \mapsto \mathcal{T}$ defined as:

$$
\operatorname{Tag}\left(\mu,\left(r_{0}, \ldots, r_{c}\right)\right):=r_{0}+r_{1} \cdot \mu+\cdots+r_{c} \cdot \mu^{c}
$$

is a c-time $\left(n, k, 2^{\frac{c n}{c+1}-k}\right)$-secure message authentication code.

Proof. Let $U$ be uniform in $\mathbb{F}^{c+1}$. For any fixed strategy of Eve, and $r \in \mathbb{F}^{c+1}$, let $f(r)$ denote $\operatorname{Adv}_{c}^{\text {Eve }}(r)$. Let $\mu_{1}, \ldots, \mu_{c+1}$ be arbitrary distinct messages in $\mathcal{M}$. By Lemma 1 , we have that for any $\sigma \in \mathcal{T}$, the probability that $\operatorname{Tag}\left(\mu_{c+1}, U\right)=\sigma$ given $\operatorname{Tag}\left(\mu_{1}, U\right), \ldots, \operatorname{Tag}\left(\mu_{c}, U\right)$ is at most $\frac{1}{|\mathbb{F}|}=2^{-n /(c+1)}$. Hence,

$$
\mathrm{E}_{r \leftarrow U}[f(r)] \leq 2^{-\frac{n}{c+1}} .
$$

Now, consider a random key $R \in \mathbb{F}^{c+1}$, such that $\mathbf{H}_{\infty}(R) \geq k$. Then

$$
\begin{aligned}
\mathrm{E}_{r \leftarrow R}[f(r)] & =\sum_{r \in \mathbb{F}^{c+1}} \operatorname{Pr}(R=r) \cdot f(r) \\
& \leq \max _{r \in \mathbb{F}^{c+1}} \operatorname{Pr}(R=r) \sum_{r \in \mathbb{F}^{c+1}} f(r) \\
& \leq 2^{-k} \cdot 2^{n} \cdot \mathrm{E}_{r \leftarrow U}[f(r)] \\
& \leq 2^{n-k} \cdot 2^{-\frac{n}{c+1}} \\
& =2^{\frac{c n}{c+1}-k}
\end{aligned}
$$

as needed. 


\section{Acknowledgements}

We would like to thank Yevgeniy Dodis whose lecture on "Randomness in Cryptography" inspired us to work on this problem. The research of the second author is supported by NSF grant 1319051.

\section{References}

[1] Carl Bosley and Yevgeniy Dodis. Does privacy require true randomness? In Theory of Cryptography, pages 1-20. Springer, 2007.

[2] Yevgeniy Dodis and Joel Spencer. On the (non)universality of the one-time pad. In Proceedings of the 43rd Annual IEEE Symposium on Foundations of Computer Science, pages 376-385. IEEE Computer Society, 2002.

[3] Serge Fehr and Stefan Berens. On the conditional Rényi entropy. Information Theory, IEEE Transactions on, 60(11):6801-6810, Nov 2014.

[4] Masahito Hayashi and Toyohiro Tsurumaru. More efficient privacy amplification with less random seeds via dual universal hash function. arXiv preprint arXiv:1311.5322, 2013.

[5] Tor Helleseth and Thomas Johansson. Universal hash functions from exponential sums over finite fields and Galois rings. In Advances in Cryptology-CRYPTO'96, pages 31-44. Springer, 1996.

[6] Douglas Stinson. Universal hashing and authentication codes. Designs, Codes and Cryptography, 4(3):369-380, 1994. 